\title{
SURROGATE-ASSISTED OPTIMIZATION FOR AUGMENTATION OF FINITE ELEMENT TECHNIQUES
}

\author{
Samineh Bagheri $^{1}$, Ulf Reinicke ${ }^{2}$, Denis Anders ${ }^{3}$ and Wolfgang Konen ${ }^{4}$ \\ ${ }^{1}$ Cologne University of Applied Sciences (TH Köln) \\ Computer Science Department, Campus Gummersbach, Germany \\ samineh.bagheri@th-koeln.de \\ ${ }^{2}$ Cologne University of Applied Sciences (TH Köln) \\ Group for Computational Mechanics and Fluid Dynamics \\ Campus Gummersbach, Germany \\ ulf.reinicke@th-koeln.de \\ ${ }^{3}$ Cologne University of Applied Sciences (TH Köln) \\ Group for Computational Mechanics and Fluid Dynamics \\ Campus Gummersbach, Germany \\ denis.anders@th-koeln.de \\ ${ }^{4}$ Cologne University of Applied Sciences (TH Köln) \\ Computer Science Department, Campus Gummersbach, Germany \\ wolfgang.konen@th-koeln.de
}

Key words: Expensive optimization, surrogate models, RBF interpolation, FEM simulation

\begin{abstract}
The application of finite element techniques for the analysis and optimization of complex thermo-mechanical structures typically involves highly nonlinear models for material characterization, tribological contact, large deformation, damage, etc. These nonlinearities usually call for a higher-order Spatio-temporal discretization, including a large number of elements and time-steps in order to provide good convergence and sufficiently accurate simulation results. This inevitably leads to many expensive simulations in terms of cost and time if an optimization or adaption of model parameters has to be done. In this work, a FEM simulation modeling approach is proposed, which uses radial basis function interpolations (RBF) as efficient surrogate models to save FEM simulations. Also, a surrogate-assisted optimization algorithm [3] is utilized to find the parameter setting, which would lead to maximum damage in a simple tensile testing scenario involving a notched specimen with as few FEM simulations as possible. The relatively high accuracy of the utilized surrogate models showcases promising results and indicates the potential of surrogate models in saving time-expensive simulations.
\end{abstract}

\section{INTRODUCTION}

As the computational power continuously grows, simulation software rapidly evolve to become more accurate and detailed but also more time-consuming to run.

Spethmann et al. in [19] give a comprehensive overview of the evolution of FEM-based crash simulators 
since their development in 1960 till today.

In many engineering fields finding an optimal design for large complex systems that are highly parametrized became popular only after developing detailed, accurate simulation software replacing real experiments. Although modern optimization heuristics $[5,20]$ are suitable to address high-dimensional derivativefree optimization problems like simulation-based optimization problems, they often demand more than affordable simulations. Surrogate-assisted optimization algorithms, aiming at reducing the number of expensive function evaluations (in our case simulations) by replacing the real function calls with cheap mathematical models are the state-of-the-art techniques in the field of efficient optimization. Although in the last years a significant amount of effort is devoted to the development and evaluation of surrogateassisted methods with the purpose of solving real-world optimization problems, most of the utilized evaluation benchmarks are synthetic $[1,9,14]$.

Bagheri et al. [3] show promising results applying a constrained surrogate-assisted optimizer on solving MOPTA08 [11] problem coming originally from the automotive industry, but simplified in different ways. They use radial basis function interpolation (RBF) as surrogates. In this work we formulate a simulation-based design optimization as a Bi-level optimization, where the goal is to find a set of parameter setting that optimizes an objective function all over a component. We also describe how to approach such problem efficiently with surrogate models in two stages. We test the described algorithm on a simple damage optimization problem.

The rest of this paper is organized as follows In Sec. 2, the damage analysis of a viscoelastic tension rod is employed as a practical example of application. Sec. 3 describes a two-staged novel method for efficiently modeling FEM simulation responses and fo
problems. After describing the experimental setup
presented in Sec. 5. A short summaty in Section 6
2 PROBLEMDESCRIPTION
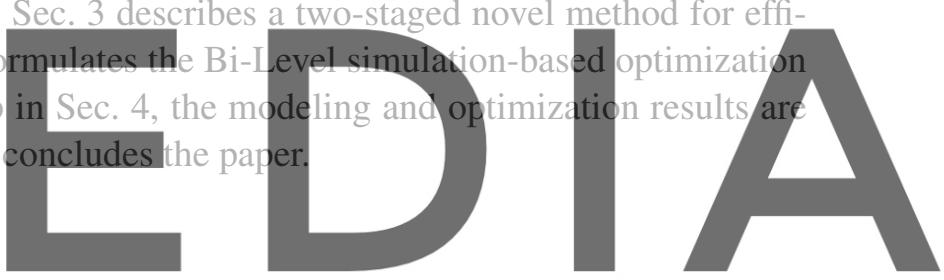

In this work, we consider a two-dimensional finite element model of a simple tensile testing scenario

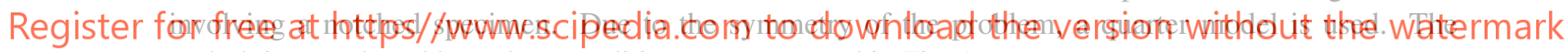
underlying mesh and boundary conditions are presented in Fig. 1.

The specimen is loaded uniformly by a time-dependent displacement function $u_{y}(t)=\frac{u_{\max }}{\tau_{\text {load }}} \cdot t$, where $u_{\max }$ and $\mathcal{T}_{\text {load }}$ denote a fixed maximum displacement of the specimen and the upper bound for the loading time-scale, respectively. By setting $u_{\max }=1 \mathrm{~mm}$ throughout all simulations and varying the time-scale $\mathcal{T}_{\text {load }}$ different local strain rates can be adjusted within the specimen depending on geometry and material behavior.

The material is supposed to show viscoelastic behavior which is characteristic for polymers $[17,16]$, rubber-like materials $[6,12]$ or biological tissue $[4,8]$.

Such materials are subjected to very pronounced time and temperature-dependent mechanical properties. Therefore, the analysis and understanding of damage mechanisms in viscoelastic materials are still an object of research. In this contribution, the dissipated mechanical energy density is employed as an indicator for accumulated damage. Therefore the damage parameter is given in $\mathrm{N} \cdot \mathrm{mm} / \mathrm{mm}^{3}$. To guarantee the comparability of the numerical results, the spatial mesh and the temporal discretization are kept constant throughout all simulations that served as input data for optimization. The necessary 

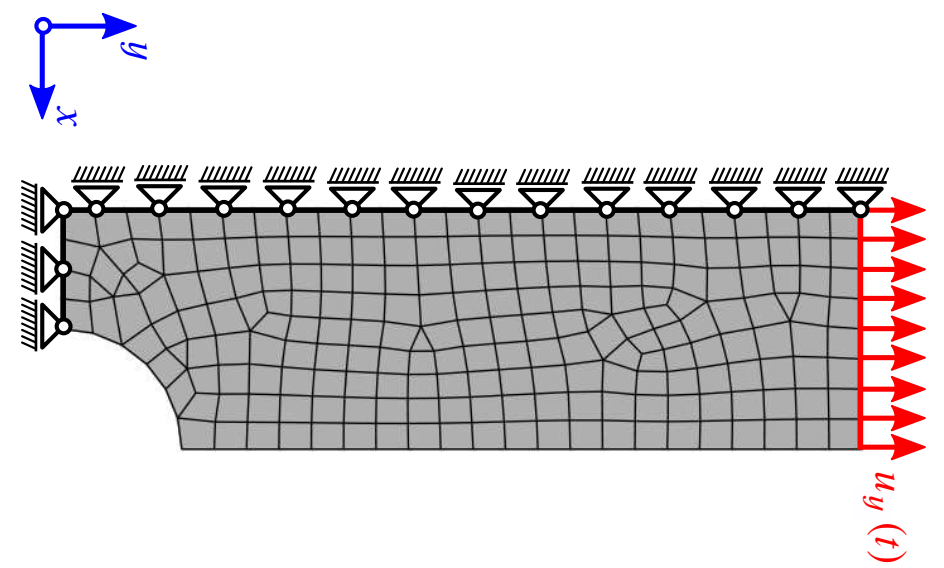

Figure 1: Illustration of the investigated quarter model involving displacement function $u_{y}(t)$ and boundary conditions

geometric structure and numerical solution of the fully coupled thermo-mechanical model equations are elaborated using the commercial finite element calculation software ABAQUS and the post-processing. In this context, ABAQUS can be regarded as a standard finite element software since it is used worldwide by engineers and researchers for various types of engineering thermo-mechanical problems, cf. [7]. In the scope of this contribution, variations of loading time-scale and temperature in each experiment are simulated, and their effect on the characteristics of damage accumulation within the specimen body is

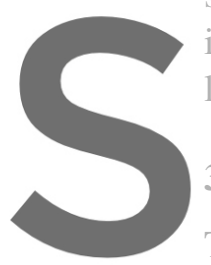
investigated. Hereby, the loading time directy affe
level has a substantial impact on creep and relaxation
3 METHODS The demanding challenge with the expensive function evaluatio
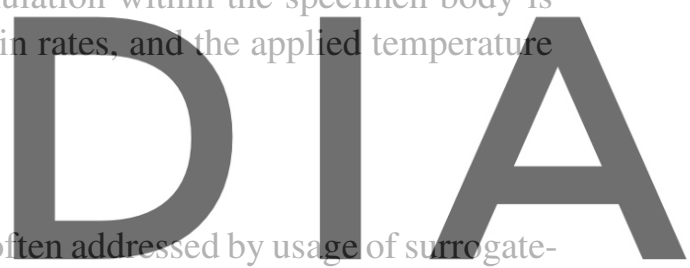
assisted algorithms. Surrogate-assisted optimizers aim at saving expensive simulation runs by replacing

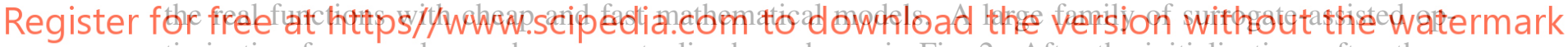
timization frameworks can be conceptualized, as shown in Fig. 2. After the initialization, often there are three main steps: modeling, optimization, and evaluation. Some surrogate-assisted optimizers are equipped with a parameter tuning block, which adjusts the optimizer's hyperparameters gradually based on the information gained about the problem in each iteration. These steps will repeat in a loop as long as the budget is not exhausted.

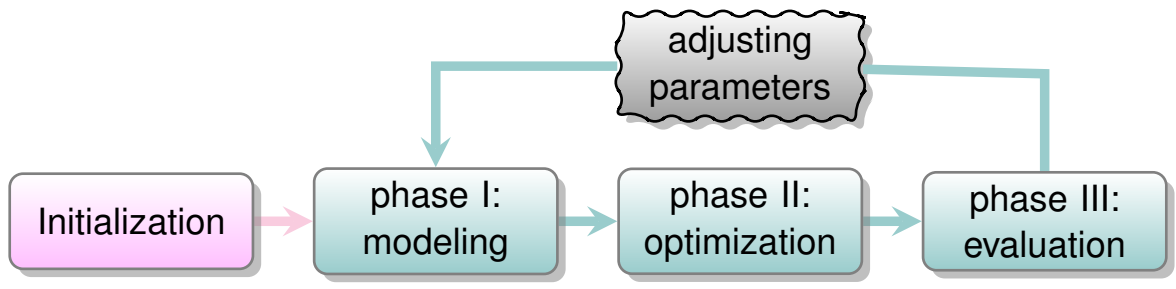

Figure 2: Surrogate-assisted optimization flowchart.

SACOBRA [3] and EGO [10] are two surrogate assisted optimizers being investigated in this work. 
SACOBRA is specifically designed for constrained optimization problems, uses RBF surrogates and benefits from self-adjusting functionalities. More details about SACOBRA can be found in [3, 2]. EGO addresses unconstrained optimization problems and uses Kriging surrogates [13]. Both algorithms can be adjusted to address Bi-level optimization problems described in 3.2.

\subsection{FEM Simulation Modeling (FSM)}

This section introduces our surrogate-assisted approach, the so-called FSM, to approximate FEM-simulation results based on very few FEM simulations. Our approach has three main phases.

phase I: building surrogate models for $n$ nodes based on the $m$ simulation results with parameter settings $\mathbf{p}_{j}$, for $j=1, \ldots, m$

phase II: predicting the objective value for a new parameter setting $\mathbf{p}_{*}$ at all $n$ nodes based on the $n$ surrogate models built during the phase I

phase IIII: fitting a model on the surface of the component based on the predicted values in phase II,

RBF interpolations are used as surrogates in phase I and phase III. It is worthy to mention that the $n$ nodes taken into account for the modeling in phase I can be a subset of all nodes. The model error can be minimized by taking the best subset of nodes into account.

\subsection{Optimizing Simulation-Based Problems}

Simulation-based optimization tasks can be formulated as Bi-level optimization problems. The goal is

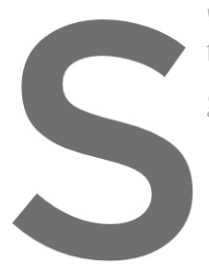

to find a design parame general a simulation-ba
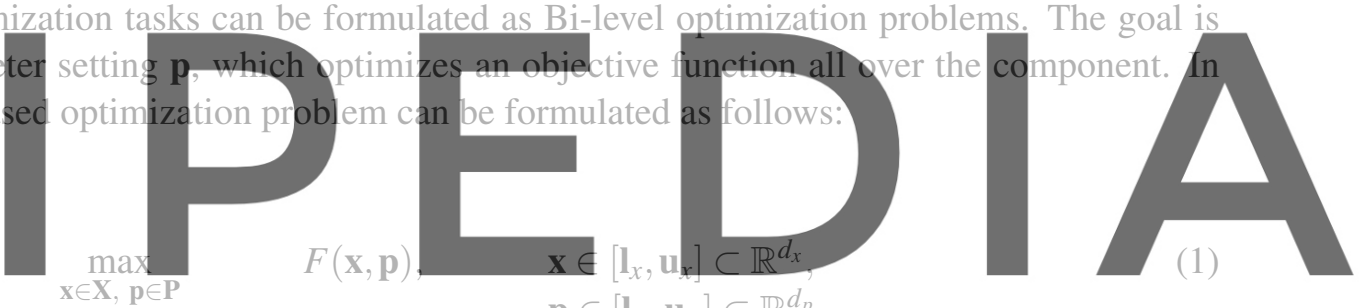

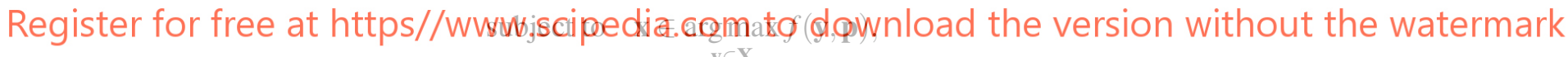

where $\mathbf{X}$ is the geometrical search space of the problem with the lower bound $\mathbf{l}_{x}$ and upper bound $\mathbf{u}_{x}$ and $\mathbf{P}$ is the parametric search space of the problem bounded between $\mathbf{l}_{p}$ and $\mathbf{l}_{p} . d_{x}$ is the dimension of the geometrical space, which is 2 for $2 \mathrm{D}$ surfaces or 3 for $3 \mathrm{D}$ spaces of the component. $d_{p}$ is the dimension of the parameter space, referring to the number of simulation parameters. The goal is to find the optimal parameter setting $p_{*}$, which maximizes function $F$ at the node where the maximum value of function $f$ is located. Maximization problems at each level can be transformed into minimization by negating the objective function without loss of generality. Expensive Bi-level optimization problems can be addressed with surrogate-assisted methods [15]. However, this work's test problem can be simplified to a more straightforward optimization problem. The node with the maximum objective value is always at a fixed location in the notch root regardless of the parameter setting's choice $\mathbf{p}$, but this is not the case all the time. Therefore, the optimization problem that we deal with in this work can be formulated as 
follows:

$$
\begin{array}{ccc}
\max _{\mathbf{p} \in \mathbf{P}} & F_{x_{*}}(\mathbf{p}), & \mathbf{p} \in\left[\mathbf{l}_{p}, \mathbf{u}_{p}\right] \subset \mathbb{R}^{d_{p}}, \\
& \mathbf{x}_{*}=\underset{\mathbf{y} \in \mathbf{X}}{\arg \max } f(\mathbf{y}, \mathbf{p}) & \forall \mathbf{p}
\end{array}
$$

\section{EXPERIMENTAL SETUP}

Every simulation of the described case study returns damage values for 240 fixed nodes on a 2D surface. The variable parameters are: loading time-scale and temperature. The loading time-scale values vary in the range of $[0.5,3.5] \mathrm{s}$ and temperature varies in the range $[80,120]^{\circ} \mathrm{C}$. Using vectorized RBF models from the SACOBRA package available on $\mathrm{CRAN}^{1}$, we can model all 240 nodes efficiently in one call.

In order to evaluate the quality of the surrogate models generated by our FSM algorithm (as described in Sec. 3.1), we make use of the relative error Eq. (4).

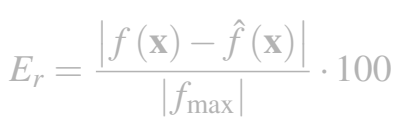

The value for $f_{\max }$ is set to 1.5 , since our experiments have shown that the damage values will not exceed this value.

The max damage optimization is

in R. The initial design

independently initialize

results are compared with

5 RESULTS
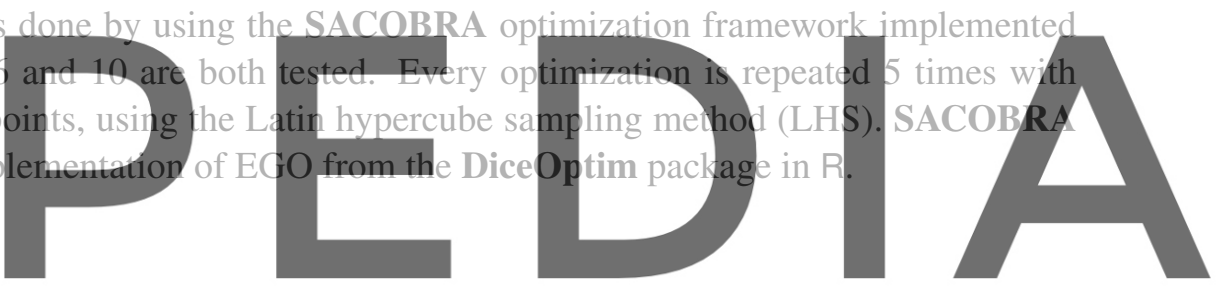

A surrogate-assisted optimizer essentially needs surrogate models which are good enough to direct the

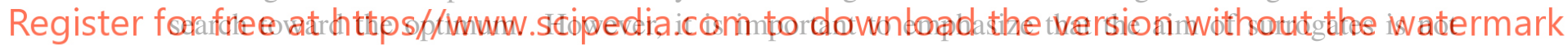
providing extremely accurate models but models which contain information about the optimum direction and can gradually improve in the interesting region.

To evaluate the quality of our surrogates, we built different models varying the size of the training set and evaluated the models on 500 unseen parameter settings. As shown in Fig. 3 the medians of model errors drop as the training size grows. However if the training population is selected completely randomly the worst case scenario errors remain as large as $4 \%$.

The large relative errors correspond to parameter settings with boundary values or parameter settings in the region with sparse samples. Identifying these regions and adding these points to the training set can improve the models' overall quality. This is being done automatically during the optimization steps with EGO as well as SACOBRA.

Fig. 4 shows the performance of different approached for maximizing the damage for the problem described in Sec. 2 for 5 independently initialized runs. The first six simulations in SACOBRA6 and EGO belong to the initialization phase. SACOBRA10 has initilization phase of ten simulations. After the

\footnotetext{
${ }^{1}$ https://cran.r-project.org/web/packages/SACOBRA
} 

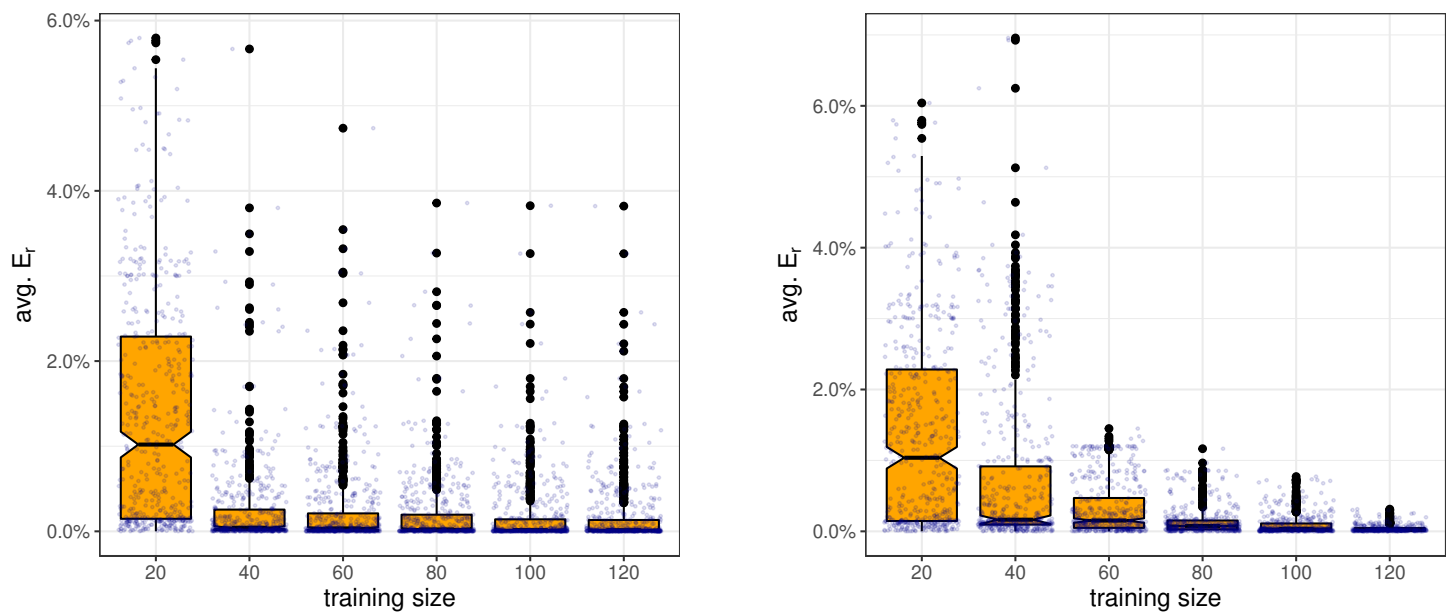

Figure 3: The relative error of the models built by means of the FSM Algorithm. The training size, meaning the number of simulations results passed to the FSM algorithm is varied and the model quality is evaluated for 500 unseen parameter settings. Left plot: in each experiment 20 randomly selected parameter settings are added to the training population. Right plot: In each experiment the 20 parameter settings, which had the largest relative error in the former experiment are added to the training population.

initialization phase, SACOBRA10 and SACOBRA6 only require four and seven more simulations to converge to the optimum, respectively. It can be shown that EGO faces an early stagnation. Moreover, one of the 5 runs with EGO crashed due to numerical instabilities and the curve shown in Fig. only related to the 4 remaini algorithm $[10,18]$.
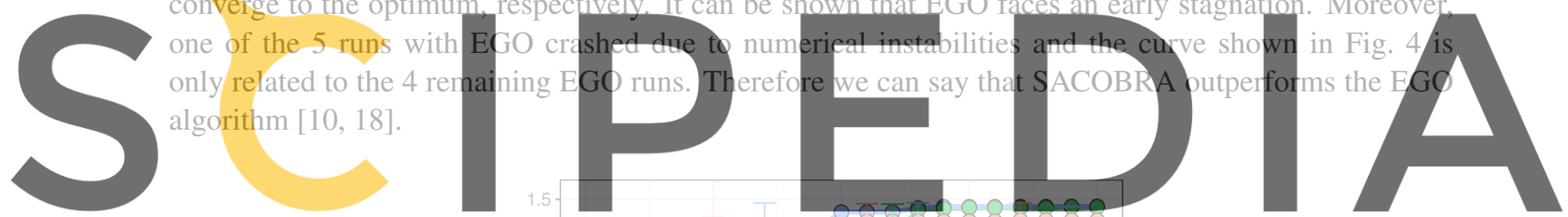

Register for free at https//www.scipedia.com to download the version without the watermark

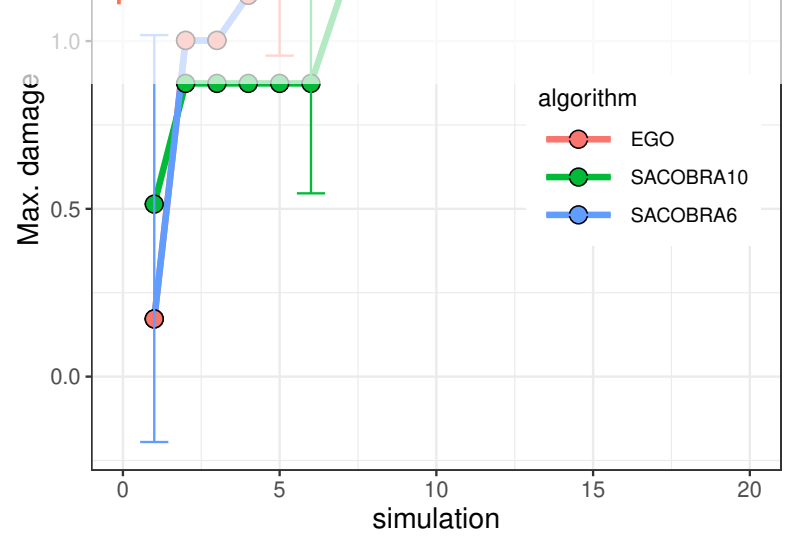

Figure 4: Optimization results for the tensile testing problem. 


\section{CONCLUSION}

The main contribution of this work is to introduce FSM, a two-staged modeling approach for efficiently modeling FEM simulations. Also we have shown that RBF surrogates can provide reasonable accuracy. We utilized a tensile testing problem as a use case and we have shown that SACOBRA outperforms EGO in terms of stability and optimization results.

For future work we are planing to take Bi-level optimization problems with more complex scenarios into account, including problems with larger parameter spaces, constrained Bi-level optimization problems and even Multi-objective Bi-level optimization problems.

\section{REFERENCES}

[1] R. Allmendinger, M. Emmerich, J. Hakanen, Y. Jin, and E. Rigoni. Surrogate-assisted multicriteria optimization: Complexities, prospective solutions, and business case. Journal of Multi-Criteria Decision Analysis, 24, 122016.

[2] S. Bagheri, W. Konen, and T. Bäck. How to solve the dilemma of margin-based equality handling methods. In Frank Hoffmann, Eyke Hüllermeier, and Ralf Mikut, editors, Proc. 28. Workshop Computational Intelligence, pages 257-270. KIT Scientific Publishing, Karlsruhe, November 2018.

[3] S. Bagheri, W. Konen, M. Emmerich, and T. Bäck. Self-adjusting parameter control for surrogateassisted constrained optimization under limited budgets. Applied Soft Computing, 61:377 - 393, 2017
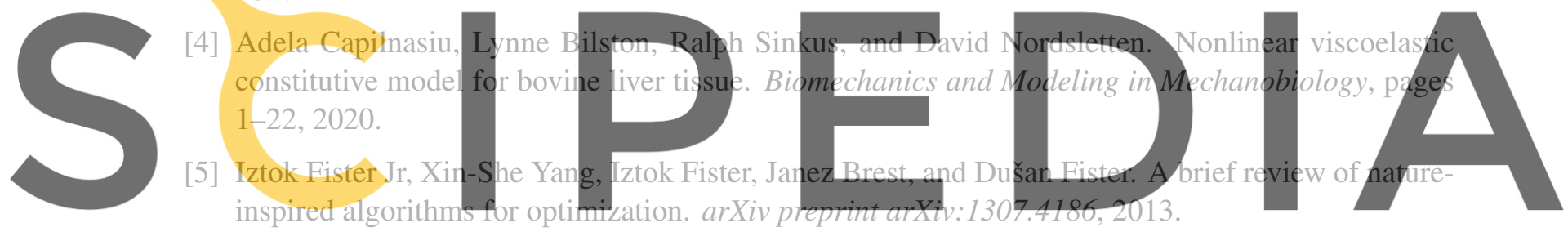

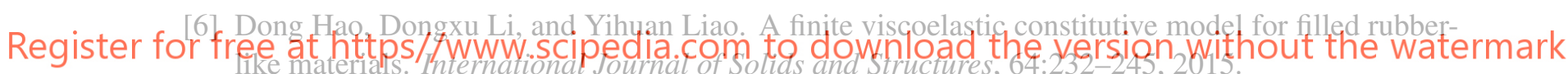

[7] S. Helwany. Applied Soil Mechanics with ABAQUS Applications. John wiley \& sons, 2007.

[8] Mohammad Hosseini-Farid, Mohammadreza Ramzanpour, Mariusz Ziejewski, and Ghodrat Karami. A compressible hyper-viscoelastic material constitutive model for human brain tissue and the identification of its parameters. International Journal of Non-Linear Mechanics, 116:147-154, 2019.

[9] Yaochu Jin. Surrogate-assisted evolutionary computation: Recent advances and future challenges. Swarm and Evolutionary Computation, 1(2):61 - 70, 2011.

[10] D. R. Jones, M. Schonlau, and W. J. Welch. Efficient global optimization of expensive black-box functions. J. of Global Optimization, 13(4):455-492, December 1998.

[11] Donald R Jones. Large-scale multi-disciplinary mass optimization in the auto industry. In MOPTA 2008 Conference (20 August 2008), 2008.

[12] H Khajehsaeid, J Arghavani, R Naghdabadi, and S Sohrabpour. A visco-hyperelastic constitutive 
model for rubber-like materials: A rate-dependent relaxation time scheme. International Journal of Engineering Science, 79:44-58, 2014.

[13] D. G. Krige. A statistical approach to some basic mine valuation problems on the witwatersrand. Journal of the Southern African Institute of Mining and Metallurgy, 52(6):119-139, 1951.

[14] Ilya Loshchilov, Marc Schoenauer, and Michele Sebag. Self-adaptive surrogate-assisted covariance matrix adaptation evolution strategy. In Proceedings of the 14th annual conference on Genetic and evolutionary computation, pages 321-328, 2012.

[15] Jesús-Adolfo Mejía-de Dios and Efrén Mezura-Montes. A surrogate-assisted metaheuristic for bilevel optimization. In Proceedings of the 2020 Genetic and Evolutionary Computation Conference, pages 629-635, 2020.

[16] A. Muliana, KR. Rajagopal, D. Tscharnuter, and G. Pinter. A nonlinear viscoelastic constitutive model for polymeric solids based on multiple natural configuration theory. International Journal of Solids and Structures, 100:95-110, 2016.

[17] Zh. Panand and Z. Liu. A novel fractional viscoelastic constitutive model for shape memory polymers. Journal of Polymer Science Part B: Polymer Physics, 56(16):1125-1134, 2018.

[18] Olivier Roustant, David Ginsbourger, and Yves Deville. Dicekriging, diceoptim: Two r packages for the analysis of computer experiments by kriging-based metamodeling and optimization. Journal of Statistical Software, Articles, 51(1):1-55, 2012.

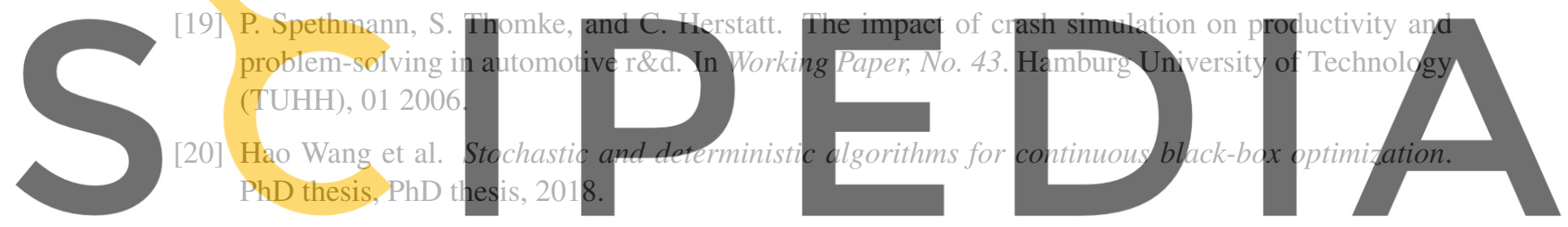

